\title{
COMPÉTENCES ET ÉCRITURES NUMÉRIQUES ORDINAIRES
}

\author{
Isabelle Cailleau, Serge Bouchardon, \\ Stéphane Crozat et Hélène Bourdeloie ${ }^{1}$
}

Les médias numériques transforment profondément les pratiques d'écriture : ils les rendent à la fois plus courantes et plus complexes. Nous posons dans cet article la question de la nature des compétences scripturales nécessaires aux « gens ordinaires » pour s'approprier ces nouveaux médias. Notre hypothèse est que l'écriture numérique requiert, au-delà d'une maîtrise de l'outil, une compréhension des spécificités du numérique. Nous défendrons cette idée en nous interrogeant sur les caractéristiques des écritures numériques des « gens ordinaires ». Puis, nous proposerons un modèle des spécificités de l'écriture numérique élaboré dans le cadre d'un projet en cours. Enfin, nous présenterons les pistes didactiques sur lesquelles nous travaillons actuellement dans le cadre de ce projet.

1 Isabelle Cailleau et Serge Bouchardon, Université de Technologie de Compiègne, EA 2223 COSTECH, Connaissance, Organisation et Systèmes TECHniques ; Stéphane Crozat, Université de Technologie de Compiègne, Unité Ingénierie des Contenus et Savoirs ; Hélène Bourdeloie, EA 2223 COSTECH, chercheuse associée LabSIC, Université Paris 1.

Recherches en communication, $\mathrm{n}^{\circ} 34$ (2010). 
Les pratiques d'écriture sont profondément transformées par les médias numériques. En effet, comme le souligne C. Barre-de Miniac (2003), « loin de détrôner l'écrit [...], les nouvelles technologies en rendent l'usage encore plus nécessaire, en multiplient et en complexifient les usages ». La pratique de l'écriture numérique est ainsi généralisée, quotidienne, ordinaire. Elle nécessite néanmoins des compétences scripturales de plus en plus complexes. Il convient donc de s'interroger sur la nature des compétences scripturales rendues nécessaires par le passage aux médias numériques (Jeanneret, 2001) ; l'enjeu étant que tout un chacun - les « gens ordinaires »-s'approprie l'usage des médias contemporains.

Dans son article sur la compétence scripturale, M. Dabène (1991) met en évidence que celle-ci suppose une connaissance et une compréhension de ce qui distingue fondamentalement l'ordre du scriptural de celui de l'oral. Ces connaissances qualifiées de « connaissances méta-scripturales » sont une composante nécessaire de la compétence scripturale $^{1}$. Dans le prolongement de ce travail, nous formulons l'hypothèse que l'écriture numérique requiert, au-delà d'une maîtrise des fonctionnalités techniques - c'est-à-dire de l'emploi de l'outil -, une connaissance et une compréhension des spécificités du numérique. Cette hypothèse se situe dans le prolongement des recherches de J. Goody (1979) pour qui tout nouveau support transforme nos manières d'agir et de connaître. Dans cette perspective, la capacité à mobiliser en situation des connaissances méta-scripturales spécifiques au numérique serait une des composantes de la compétence scripturale contemporaine. Nous défendrons cette idée en nous intéressant, dans un premier temps, aux caractéristiques des écritures numériques des « gens ordinaires ». Dans un second temps, nous décrirons les spécificités de l'écriture numérique selon un modèle élaboré dans le cadre d'un projet, intitulé «PRECIP » ${ }^{2}$, sur lequel nous travaillons actuellement. Enfin,

1 Selon M. Dabène (1991, p. 13), l'ordre du scriptural peut se caractériser par un ensemble de spécificités (matérielles, linguistiques, sociologiques, anthropologiques et psychologiques). " La maîtrise des savoir-faire que requiert la pratique scripturale passe par l'acquisition de connaissances méta-scripturales [...]», « métascripturales » au sens où ces connaissances portent sur ces spécificités de l'ordre du scriptural.

2 Le projet PRECIP (PRatiques d'ÉCriture Interactive en Picardie) est un projet de recherche financé par la Région Picardie sur les pratiques d'écriture numérique impliquant des chercheurs en sciences humaines et en sciences de l'ingénieur de l'Université de Technologie de Compiègne et de l'Université de Picardie Jules- 
nous proposons de présenter des pistes didactiques qui constituent ici un travail en cours d'élaboration.

\section{Les écritures numériques des « gens ordinaires »}

\section{Des actes d'écriture « (extra-)ordinaires »}

Les écrits des « gens ordinaires » font l'objet d'enquêtes ethnologiques depuis les années 80 . Les recherches menées sur ces pratiques ordinaires, jusqu'alors peu étudiées, révèlent le caractère déterminant de la compétence scripturale dans une culture de l'écrit. Comme le souligne D. Fabre (Fabre et al. 1997, p. XI), « l'écriture n'est pas une compétence extérieure, un savoir-faire facultatif, une injonction discontinue. Sa présence est forcément centrale et active, aucun espace ne lui est étranger $\gg$.

En mettant en évidence l'omniprésence sociale de ces écritures ordinaires, ces auteurs en viennent à tracer une frontière nette entre écrits ordinaires et écrits littéraires. Toutefois, M. Dabène (1991) remet en question la pertinence de cette opposition. En effet, dès que l'on entre dans l'ordre du scriptural - dans l'univers de l'écrit au sens large -, on s'inscrit dans une pratique culturelle ritualisée qui n'a rien d'ordinaire. On sort ainsi d'un « usage naturel du langage » (ibid.) pour entrer dans un usage par définition normé (au niveau graphique, syntaxique, générique, etc.), ritualisé, même pour les écrits les plus ordinaires. En ce sens, tout écrit est « (extra)-ordinaire », qu'il ait une finalité littéraire ou non. C'est pourquoi, communiquer par écrit suppose de connaître et de s'approprier les spécificités de cette forme de communication. Une maîtrise des « règles de transcription graphique » (ibid.) ne saurait donc suffire tant pour les professionnels que pour les $«$ gens ordinaires $\rangle^{1}$. La « connaissance méta-scripturale » de ce qui caractérise l'ordre de l'écrit est une composante nécessaire de la compétence scripturale.

Des connaissances « méta-scripturales » spécifiques au numérique

Verne. Il s'agit d'un projet en cours (2009-2012). Site internet : http://precip.fr

1 A la suite de M. Dabène, nous réserverons donc le qualificatif d' '« ordinaire » aux personnes qui produisent des écrits - les « gens ordinaires »- (par opposition aux professionnels), et ne l'utiliserons pas pour qualifier les écrits qui sont par nature «(extra)-ordinaires ». 
De plus, les spécificités de l'ordre de l'écrit nous semblent devoir être réinterrogées dès lors que son support d'inscription devient numérique. En effet, de la même manière que la culture de l'écrit a transformé nos capacités cognitives ainsi que l'a démontré J. Goody (1979), le numérique devrait entraîner une transformation de nos modes de pensée. C'est l'hypothèse défendue par B. Bachimont : « Si l'écriture a donné lieu à une " raison graphique », le numérique doit donner lieu à une « raison computationnelle » : le calcul comme technique de manipulation de symboles entraîne un mode spécifique de pensée, qui ne remplace pas les autres, mais les reconfigure » $(2000$, p. 3).

Le changement de support (des supports statiques comme le papier au support dynamique du numérique) nous paraît en effet être de nature suffisamment profonde pour justifier la remise en cause des connaissances méta-scripturales. Ainsi, pour écrire sur un support numérique, il ne s'agit plus seulement de posséder les connaissances méta-scripturales liées aux supports traditionnels mais également des connaissances complémentaires, voire alternatives, liées aux propriétés qui définissent le numérique.

\section{Des connaissances « méta-scripturales » aux compétences scripturales}

La question qui guide ce travail est ainsi celle de l'identification des spécificités qui caractérisent l'ordre du scriptural à l'ère du numérique, l'enjeu étant de caractériser une des composantes de la compétence scripturale contemporaine.

A la suite de P. Perrenoud (2004a), nous définissons la compétence comme « un pouvoir d'agir [...] dans une classe de situations comparables ». Et, comme il le souligne, cette capacité d'action n'exclut pas le savoir. " Bien au contraire, détenir certains savoirs est la condition d'une action efficace. [...] Aucune action ne peut atteindre ses fins sans une connaissance du système dont elle tente de prendre le contrôle, qu'il soit humain, matériel, symbolique ou les trois à la fois. »

Nous faisons alors l'hypothèse suivante : pour agir efficacement dans des situations d'écriture numérique, une connaissance des spécificités théoriques, applicatives et interprétatives de ces dispositifs est nécessaire. La compréhension de l'articulation entre ces trois dimensions est selon nous une connaissance méta-scripturale déterminante pour l'écriture numérique. 
Toutefois, poser la nécessité de cette connaissance ne va pas de soi. Les outils numériques étant de plus en plus transparents et intuitifs d'usage, il pourrait même sembler qu'au contraire une compétence opératoire suffise. Cependant, lorsque qu'une situation problématique se présente, les connaissances pour poser ce problème correctement et le résoudre efficacement manquent souvent. Le tâtonnement qui s'en suit permet rarement un agir efficace. Nous avons par exemple observé des élèves ingénieurs ne pas savoir gérer un copier-coller de texte d'une application à une autre dont le résultat était illisible. Ils ne détiennent en effet pas les connaissances qui leur permettraient de comprendre que même si le numérique permet techniquement une duplication parfaite, l'encodage de l'information varie selon les applications. Le texte qu'ils lisent n'est pas celui stocké en mémoire et par conséquent, si une application ne gère par la conversion, le résultat sera illisible. Cette connaissance leur permettrait de poser le problème et donc de mettre en place des stratégies efficaces.

Il ne suffit pas pour autant de détenir cette connaissance, il faut également savoir la mobiliser, se servir de cette ressource à bon escient ce qui va requérir « un travail spécifique de formation, qui s'apparente à un entraînement réflexif »(Perrenoud, 2004a). Les choix de scénarisation pédagogiques ( $c f .3^{\mathrm{e}}$ partie) joueront donc un rôle déterminant pour la mobilisation de la connaissance comme ressource effective de la compétence.

Il serait toutefois possible d'objecter que la plupart des situations d'écriture numérique ne relèvent pas de situations posant problème. Nous sommes néanmoins tous les jours confrontés à des environnements d'écriture numérique de plus en plus diversifiés et évolutifs. Ces environnements portent des visions de l'écriture numérique, ils relèvent d'une " énonciation éditoriale " (Souchier, Jeanneret, 2005). Des choix ont été opérés au sein des possibles ouverts par les propriétés du support numérique et ces choix ne sont absolument pas neutres. Il nous semble alors qu'il existe un enjeu fort à savoir et à comprendre que nous manipulons des applications, des « architextes » (Jeanneret, 2001), qui formatent nos pratiques d'écriture et qui vont prescrire sans les déterminer des pratiques interprétatives auctoriales et lectoriales. À titre d'illustration, prendre conscience des choix éditoriaux liés à la présentation de soi au sein d'un réseau, qui sont par exemple inscrits dans le formulaire de saisie du profil de Facebook, devrait conduire à mettre en place une stratégie d'écriture plus réfléchie, notamment dans le choix des informations renseignées et/ou publiées. Les connaissances 
méta-scripturales spécifiques au numérique seraient ainsi également la condition d'une compétence critique ayant un impact sur la compétence scripturale.

Afin de tenter de cerner ces nouvelles connaissances méta-scripturales, nous avons mené, dans le cadre du projet PRECIP, un travail visant à identifier les propriétés du numérique (ou des propriétés, sans avoir donc une ambition d'exhaustivité), en particulier celles mobilisées dans le cadre des pratiques d'écriture numérique.

\section{Les propriétés du média numérique appréhendées}

\section{à travers trois niveaux}

Nous proposons ici un modèle à finalité heuristique visant à fournir une représentation des propriétés du numérique, et plus particulièrement de l'écriture numérique. Ce travail est le résultat de la première phase du projet PRECIP. Ce projet a en effet pour objectif de former des « gens ordinaires »-en particulier des collégiens et des lycéens -, aux pratiques d'écriture numérique. Il n'est donc pas question de former aux outils (bureautique, graphisme, multimédia, etc.) mais aux connaissances « méta » qui rendent possible cette écriture et de travailler à leur mobilisation en situation d'écriture. Afin de réaliser ce programme de formation à l'écriture numérique, la première phase a consisté à recenser et organiser les propriétés fondamentales et les formes d'expression propres ou reconfigurées par le numérique (écriture hypertextuelle, multimédia, collaborative, etc.).

Cette formalisation s'appuie sur l'approche théorique du numérique définie par B. Bachimont (2007). Notre démarche est ainsi la conséquence d'un positionnement théorique qui pose que les pratiques se développent à partir de possibilités techniques et que le support matériel joue un rôle dans la structuration de la connaissance. Cette démarche a abouti à une description du numérique selon trois niveaux : théorique, applicatif et interprétatif. La déclinaison de ces trois niveaux a vocation à nous fournir une représentation simplifiée, un modèle des spécificités de l'écriture numérique exploitable sur un plan pédagogique.

Il est manifeste que toute entreprise de définition des propriétés du numérique est une tâche non seulement délicate mais aussi infinie, en raison de la multiplicité des possibilités apportées par ce support mais aussi de sa constante évolution. Aussi deux précautions méthodolo- 
giques s'imposent. D'une part, la démarche ne recherche pas l'exhaustivité : la modélisation, nécessairement simplificatrice, est ici orientée par sa finalité pédagogique. D'autre part, ce modèle n'a aucune prétention à l'universalité mais vise à faire état d'un point de vue permettant de rendre intelligible les spécificités du numérique dans une perspective d'enseignement.

\section{Le niveau théorique}

Le support numérique se caractérise par deux principes essentiels : il est fondé sur la discrétisation (unités d'information indépendantes) et la manipulation, qui consiste en un calcul opéré sur ces unités discrètes. Ces dernières sont dépourvues de sémantique a priori et leur codage, en 0 et 1 (code binaire), est arbitraire. L'accès au sens n'est possible qu'après transformation par des fonctions de calcul (algorithmes) sur le code binaire, fonctions qui donnent lieu à des formes sémiotiques interprétables par l'homme. Ces transformations relèvent du second niveau (applicatif) tandis que le résultat de ces transformations, interprétable par un sujet, relève du troisième niveau (interprétatif).

Dans cette optique, la maîtrise théorique du numérique suppose la compréhension des propriétés du niveau 1, qui relèvent de principes fondamentaux du numérique conditionnant ${ }^{1}$ les possibles et les modalités de fonctionnement profondes aux niveaux 2 et 3 . Autrement dit, les propriétés des niveaux 2 et 3 ne sont possibles que parce que le numérique repose sur deux principes - discrétisation et manipulation - qui se déclinent en six propriétés fondamentales de niveau 1 : la manipulabilité, l'abstraction, l'adressabilité, l'intégration, la duplication parfaite et l'interconnexion ${ }^{2}$. Ces propriétés n'en restent pas moins abstraites par définition, non observables empiriquement et ne s'appréhendent qu'à travers les niveaux 2 et 3 qui les actualisent.

1 Il ne s'agit pas ici d'un déterminisme technique. Nous reprenons en effet la thèse de B. Bachimont (2004, p. 119) pour qui, « de manière générale, la structure physique et matérielle de l'inscription surdétermine et conditionne son interprétation, mais ne la programme ni ne la détermine à l'avance ».

2 Ces propriétés ont été représentées sous forme d'un graphe, régulièrement remis à jour de manière collaborative en fonction de l'avancée de nos travaux. Il est consultable à l'adresse suivante : http://www.utc.fr/si28/precip/wp-content/ uploads/2010/07/PRECIP-les-3-niveaux-du-numerique.pdf 


\section{Le niveau applicatif}

Le second niveau est un formatage technique du premier niveau. Le code binaire est transformé par les calculs opérés sur lui en vue d'obtenir des contenus (textes, sons, images, etc.) et des fonctionnalités manipulables (applications logicielles). Il introduit donc une sémantique au sens où les applications offrent des fonctionnalités qui, d'emblée, proposent, voire imposent des choix quant à la manière de manipuler le matériau numérique (contenus) et d'interagir avec lui. Les principes techniques s'actualisent ainsi en fonction de transformations (niveau 2) qui - commandées par l'interaction avec l'humain - produisent des formes sémiotiques interprétables (niveau 3).

Par exemple, l'adressabilité - correspondant au fait que toute unité d'information possède une adresse -, s'actualise dans la fonction d'indexation qui permet d'associer des métadonnées (par exemple des commentaires dans un traitement de texte) à n'importe quelle partie d'un document numérique, y compris le mot d'un texte ou l'image d'une vidéo.

Le niveau 2 permet donc d'actualiser et de manipuler les principes du niveau 1, par la matérialisation qu'il en propose d'une part, et par le codage de fonctionnalités qui repose sur des choix parmi les possibles ouverts par le niveau 1 d'autre part. Néanmoins, même si le niveau 2 restreint les possibles du niveau 1 (par l'ensemble de fonctionnalités applicatives qu'il propose), le niveau 2 reste fortement combinatoire : son exploitation au niveau des pratiques (niveau 3) pouvant prendre des formes multiples (allant jusqu'au détournement). En effet, le champ fonctionnel ouvert par le niveau 2 ne produit du sens que via certaines utilisations des fonctions qui se concrétisent au niveau interprétatif (niveau 3).

\section{Le niveau interprétatif}

Le troisième niveau correspond donc à la " fabrique " effective du sens par l'activation de certaines fonctions selon certaines modalités. Ces modalités sont en particulier dépendantes des contenus euxmêmes : chaque contenu (texte, image, son, vidéo, etc.) renferme une logique sui generis qui conduit à une certaine inertie, entendue comme une résistance lors des manipulations. L'usage d'une application résulte donc toujours d'un compromis entre les possibilités fonctionnelles du numérique et l'inertie propre au contenu. Il est par exemple technique- 
ment possible de segmenter un texte à n'importe quel endroit (une lettre peut être coupée en deux dans un logiciel de traitement d'image). Sur le plan sémiotique toutefois, une telle segmentation ne fait a priori pas sens.

En somme, le niveau théorique définit les potentialités techniques du support d'un point de vue théorique, le niveau applicatif caractérise ce qu'il est possible de faire avec ce support et le niveau interprétatif ajoute du sens aux potentialités applicatives.

\begin{tabular}{|c|c|c|}
\hline Niveau 1 & Niveau 2 & Niveau 3 \\
\hline Manipulabilité & Transformation & $\begin{array}{l}\text { Raffinement progressif } \\
\text { de l'écriture }\end{array}$ \\
\hline \multirow[t]{3}{*}{$\begin{array}{l}\text { Toute information numé- } \\
\text { rique est codée en binaire. } \\
\text { Elle peut être manipulée } \\
\text { par un algorithme. }\end{array}$} & $\begin{array}{l}\text { Toute information peut } \\
\text { être transformée en une } \\
\text { autre par un automate (ex : } \\
\text { « Remplacer par »). }\end{array}$ & $\begin{array}{l}\text { Le passage du brouillon } \\
\text { au document finalisé s'ef- } \\
\text { fectue sans rupture maté- } \\
\text { rielle. }\end{array}$ \\
\hline & Interactivité & Introduction de données \\
\hline & $\begin{array}{l}\text { L'information peut être } \\
\text { manipulée par l'utilisateur } \\
\text { suivant des programmes } \\
\text { établis (ex : navigation } \\
\text { dans les chapitres d'un } \\
\text { DVD-Rom). }\end{array}$ & $\begin{array}{l}\text { Il est possible de modi- } \\
\text { fier dynamiquement un } \\
\text { contenu en fonction de } \\
\text { données entrées par l'utili- } \\
\text { sateur (ex : formulaire). }\end{array}$ \\
\hline Abstraction & Modélisation & Écriture sous modèle \\
\hline $\begin{array}{l}\text { Le fait de coder en binaire } \\
\text { crée une coupure matérielle } \\
\text { par rapport au substrat } \\
\text { physique et une coupure } \\
\text { sémantique par rapport au } \\
\text { contenu produit. }\end{array}$ & $\begin{array}{l}\text { Il est possible d'inscrire } \\
\text { dans un logiciel des règles } \\
\text { pour contrôler l'écriture } \\
\text { (ex : remplir obligatoire- } \\
\text { ment le champ " nom " } \\
\text { dans un formulaire). }\end{array}$ & $\begin{array}{l}\text { Les suites bureautiques } \\
\text { proposent des " organisa- } \\
\text { tions type » selon le type } \\
\text { de document visé (ex : CV, } \\
\text { lettre, diaporama, etc.). }\end{array}$ \\
\hline Adressabilité & Indexation & 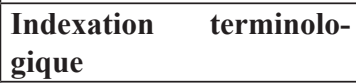 \\
\hline $\begin{array}{l}\text { Toute information codée } \\
\text { possède une adresse qui } \\
\text { permet d'y accéder. }\end{array}$ & $\begin{array}{l}\text { Il est possible de poser des } \\
\text { ancres dans un contenu et } \\
\text { d'y associer des métadon- } \\
\text { nées. }\end{array}$ & $\begin{array}{l}\text { Il est possible d'indexer les } \\
\text { termes significatifs d'un } \\
\text { document pour créer un } \\
\text { index. }\end{array}$ \\
\hline Intégration & Multimédia & Écriture multimédia \\
\hline
\end{tabular}




\begin{tabular}{|c|c|c|}
\hline $\begin{array}{l}\text { Il est possible d'encoder } \\
\text { n'importe quel format } \\
\text { (caractères alphabétiques, } \\
\text { sons, images, etc.) et de les } \\
\text { représenter sur un support } \\
\text { unique (écran). }\end{array}$ & $\begin{array}{l}\text { Les applications permettent } \\
\text { de combiner plusieurs } \\
\text { médias au sein d'un même } \\
\text { document. }\end{array}$ & $\begin{array}{l}\text { Il est possible par exemple } \\
\text { d'insérer une vidéo et du } \\
\text { texte sur une diapositive de } \\
\text { diaporama. }\end{array}$ \\
\hline Duplication (parfaite) & Historisation & Versionnage \\
\hline $\begin{array}{l}\text { Il est possible de dupli- } \\
\text { quer n'importe quelle unité } \\
\text { d'information. Les infor- } \\
\text { mations sélectionnées et } \\
\text { dupliquées le seront sans } \\
\text { perte. }\end{array}$ & $\begin{array}{l}\text { Il est possible de conserver } \\
\text { un historique de chaque } \\
\text { modification d'un docu- } \\
\text { ment. }\end{array}$ & $\begin{array}{l}\text { Il est possible de définir des } \\
\text { règles pour sélectionner les } \\
\text { versions qui méritent d'être } \\
\text { conservées. }\end{array}$ \\
\hline Interconnexion & Contribution en ligne & Méta-discursivité \\
\hline $\begin{array}{l}\text { Manipulable et adressable, } \\
\text { l'information numérique } \\
\text { peut être reliée à une multi- } \\
\text { plicité d'autres points } \\
\text { lorsqu'elle est inscrite au } \\
\text { sein d'une architecture en } \\
\text { réseau. }\end{array}$ & $\begin{array}{l}\text { Il est possible de permettre } \\
\text { aux utilisateurs de modifier } \\
\text { ou de produire un contenu } \\
\text { en ligne (Web 2.0). }\end{array}$ & $\begin{array}{l}\text { Un lecteur de blog peut } \\
\text { poster des commentaires. }\end{array}$ \\
\hline
\end{tabular}

Table 1 : exemples extraits du graphe du projet PRECIP « Les 3 niveaux du numérique».

Notre position consiste à considérer que les pratiques d'enseignement des outils (notamment en bureautique) abordent majoritairement le niveau 3, rarement le niveau 2 et jamais le niveau 1. Ces enseignements du niveau 3 permettent d'accéder à des savoir-faire procéduraux efficaces dans un environnement familier. Ils ne permettent pas, comme nous l'avons souligné plus haut, un agir efficace face à une situation posant problème. Ils permettent encore moins un usage critique des dispositifs d'écriture, ce dernier supposant une réflexion sur les choix opérés au niveau 2 en tant qu'ils sont une sélection au sein des possibles de niveau 1.

À l'aune de ces considérations, nous pensons qu'il existe un enjeu fort à enseigner la théorie du numérique (niveau 1) et c'est pourquoi le modèle que nous avons formalisé et qui caractérise le numérique à travers trois niveaux nous paraît être un outil pertinent pour élaborer des séquences pédagogiques intégrant cette dimension théorique. Nous nous proposons donc d'illustrer les modalités possibles pour un enseignement du numérique théorique au travers de quelques exemples. 


\section{Quelques pistes didactiques}

L'enseignement de ce modèle heuristique des spécificités de l'écriture numérique est l'objectif de la seconde phase du projet PRECIP. Aussi, nos travaux en cours consistent-ils à élaborer des séquences pédagogiques destinées à être expérimentées auprès de collégiens et de lycéens. Nous proposons donc de présenter dans cette dernière partie notre approche en l'illustrant par deux exemples ciblés sur un public de lycéens.

\section{Présentation de la démarche}

L'articulation du modèle en trois niveaux nous a conduit à imaginer un scénario pédagogique générique (déclinable selon différentes approches pédagogiques nommées "modules-type »). La finalité de ce scénario est de permettre l'acquisition des connaissances méta-scripturales visées par une découverte et une appropriation de la logique des trois niveaux en vue de sa mobilisation en situation d'écriture comme composante des compétences scripturales.

À titre d'exemple, le module-type A illustré ci-dessous (Figure 1) se compose d'une sous-séquence de découverte qui articule un questionnement sur les pratiques antérieures et les apports théoriques ainsi qu'une sous-séquence de mise en situation de production réelle. Cette mise en situation est conçue de manière à permettre de provoquer la mobilisation des connaissances découvertes et d'évaluer le degré d'appropriation de la propriété de niveau 2 étudiée par les apprenants. La séquence de " découverte » se compose des activités pédagogiques suivantes :

- Une présentation de la propriété de niveau 2 choisie pour le module (durée : 5 minutes) : il s'agit ici de poser l'objectif général des activités qui vont suivre en introduisant brièvement la propriété étudiée (par exemple l'hypertextualisation) et ses enjeux.

- Une mise en situation (durée : 45 minutes) : un exercice dont la consigne est conçue pour permettre lors de la manipulation d'une application de produire des écrits et d'identifier des problèmes liés à la propriété en question qui pourront faire l'objet d'une analyse critique à l'étape suivante. 
- Une prise de conscience des spécificités de la notion de niveau 2 étudiée : l'enseignant sélectionne une ou deux productions représentatives (c'est-à-dire comportant des erreurs courantes susceptibles d'être mises en évidence) et anime leur analyse critique en groupe. La stratégie consiste ici à mettre en relief des « cas d'usage limites » pour générer une prise de conscience des contraintes induites par la propriété étudiée.

- Une explication des conditions de possibilité de la notion de niveau 2 étudiée par le niveau théorique (niveau 1) correspondant : il s'agit ici d'expliquer aux élèves quelle est la propriété du numérique théorique qui rend possible les fonctions observées au niveau applicatif (par exemple l'adressabilité pour l'hypertextualisation).

- Une illustration des implications au niveau interprétatif (niveau 3) : ayant compris pourquoi le numérique permettait de disposer de certaines fonctionnalités au niveau applicatif, il s'agit alors de présenter aux élèves une palette représentative d'actualisation de ces fonctionnalités dans les pratiques (pour l'hypertextualisation, il pourrait s'agir d'illustrer la diversité des figures de manipulation possibles (Bouchardon, 2007) auxquelles elle donne lieu).

Cette première séquence doit ainsi permettre aux élèves de comprendre l'articulation des trois niveaux pour la propriété de niveau 2 étudiée, et donc d'acquérir les connaissances méta-scripturales associées. Il s'agit alors, dans une seconde séquence, de susciter une mobilisation de cet acquis par une production réelle dans le cadre d'une mise en situation. Cette dernière doit permettre une évaluation de la compétence au sens de Perrenoud (2004b), autrement dit être conçue de manière à ce que la mobilisation de la connaissance acquise précédemment constitue une ressource efficace pour résoudre un problème complexe et qu'elle contribue au renforcement de la compétence. 


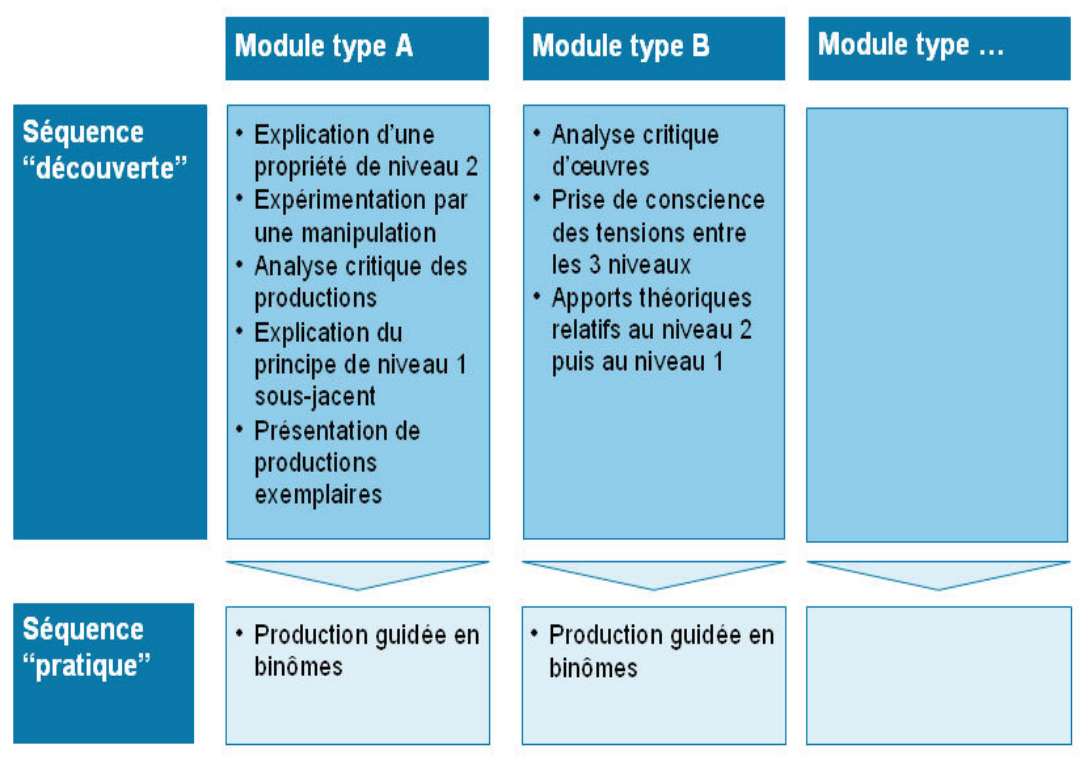

Figure 1 : scénario pédagogique générique. 
Les tables 2 et 3 présentent deux exemples de la séquence « découverte » pour le module «type $A$ » : écriture sous modèle et intersémiotisation.

\begin{tabular}{|c|c|c|c|c|}
\hline Type & Durée & Intitulé & Objectif & Ressources \\
\hline Niveau 2 & 5 , & Multimédia & Expliquer la propriété & Support expositif \\
\hline \multirow{3}{*}{$\begin{array}{l}\text { Mise en } \\
\text { situation }\end{array}$} & \multirow{3}{*}{$45^{\prime}$} & $\begin{array}{l}\text { Illustrer un discours } \\
\text { oral }\end{array}$ & \multirow{3}{*}{ Faire manipuler } & Déroulé d'exercice \\
\hline & & $\begin{array}{l}\text { 1. Prendre connais- } \\
\text { sance d'un discours } \\
\text { oral chapitré }\end{array}$ & & $\begin{array}{l}\text { Chaîne éditoriale } \\
\text { Scenari/Webradio } 1\end{array}$ \\
\hline & & $\begin{array}{l}\text { 2. Associer des } \\
\text { images ou des textes } \\
\text { aux chapitres }\end{array}$ & & $\begin{array}{l}\text { Émission } 2000 \text { ans } \\
\text { d'histoire chapitrée ; } \\
\text { dossier d'images et de } \\
\text { Textes }\end{array}$ \\
\hline Rupture & $30^{\prime}$ & $\begin{array}{l}\text { Analyser les docu- } \\
\text { ments enrichis par les } \\
\text { élèves }\end{array}$ & $\begin{array}{l}\text { Souligner des } \\
\text { problèmes d'asso- } \\
\text { ciation } \\
\text { - texte trop long par } \\
\text { rapport au temps } \\
\text { d'affichage, } \\
\text { - séquence d'image } \\
\text { inappropriée, } \\
\text { - mauvaise adéquation } \\
\text { image/texte } \\
\text {-... }\end{array}$ & $\begin{array}{l}\text { Observations atten- } \\
\text { dues }\end{array}$ \\
\hline Niveau 1 & $30^{\prime}$ & Intégration & Expliquer la propriété & Support expositif \\
\hline Niveau 3 & $10^{\prime}$ & Intersémiotisation & $\begin{array}{l}\text { Montrer des exemples } \\
\text { pertinents }\end{array}$ & $\begin{array}{l}\text { Émissions INA/ } \\
\text { GRM }^{1}\end{array}$ \\
\hline
\end{tabular}

Table 2 : exemple pour la notion de niveau 2 «multimédia ».

1 Émissions consultables sur le site du Groupe de Recherches Musicales (GRM) : http://www.ina-entreprise.com/entreprise/activites/recherches-musicales/webradio. html 


\begin{tabular}{|c|c|c|c|c|}
\hline Type & Durée & Intitulé & Objectif & Ressources \\
\hline Niveau 2 & $5^{\prime}$ & $\begin{array}{l}\text { Modélisation du } \\
\text { contenu }\end{array}$ & Introduire la propriété & Support expositif \\
\hline \multirow{3}{*}{$\begin{array}{l}\text { Mise en } \\
\text { situation }\end{array}$} & \multirow{3}{*}{$45^{\prime}$} & $\begin{array}{l}\text { Préparer une présen- } \\
\text { tation assistée par } \\
\text { ordinateur }\end{array}$ & \multirow{3}{*}{$\begin{array}{l}\text { Expérimenter des } \\
\text { contraintes : } \\
\text { - du format diaposi- } \\
\text { tive (page } v s \text { écran) } \\
\text { - de la séquentialité } \\
\text { d'une présentation }\end{array}$} & \multirow{3}{*}{$\begin{array}{l}\text { Support rédigé de } \\
\text { base et consignes }\end{array}$} \\
\hline & & $\begin{array}{l}\text { 1. Transposer un } \\
\text { contenu rédigé } \\
\text { (exposé) }\end{array}$ & & \\
\hline & & $\begin{array}{l}\text { 2. Insérer des liens } \\
\text { hypertexte dans les } \\
\text { diapositives }\end{array}$ & & \\
\hline Rupture & $30^{\prime}$ & Exposé/débat & $\begin{array}{l}\text { Commenter les } \\
\text { contraintes des } \\
\text { formats diaposi- } \\
\text { tives et du caractère } \\
\text { séquentiel d'un } \\
\text { diaporama } \\
\text { - contenus support de } \\
\text { l'oral (peu rédigés, } \\
\text { non autonomes, sous } \\
\text { forme de liste et } \\
\text { schémas) } \\
\text { - difficulté d'une } \\
\text { navigation hyper- } \\
\text { textuelle dans un } \\
\text { contenu prévu pour } \\
\text { être séquentiel }\end{array}$ & $\begin{array}{l}\text { Liste des erreurs } \\
\text { courantes }\end{array}$ \\
\hline Niveau 1 & $30^{\prime}$ & Abstraction & $\begin{array}{l}\text { Comprendre la } \\
\text { propriété et son } \\
\text { impact sur le niveau } 2\end{array}$ & Support expositif \\
\hline Niveau 3 & $10^{\prime}$ & $\begin{array}{l}\text { Écriture contrôlée par } \\
\text { la Machine }\end{array}$ & $\begin{array}{l}\text { Montrer un exemple } \\
\text { dans un système plus } \\
\text { Contraignant }\end{array}$ & $\begin{array}{l}\text { Chaîne documen- } \\
\text { taire éditoriale, } \\
\text { modèles Bureautiques } \\
(\text { OPTIM })^{1}\end{array}$ \\
\hline
\end{tabular}

Table 3 : exemple pour la notion de niveau 2 « écriture sous modèle »

Ce « module de type A » est fondé sur une prise de conscience des limites des pratiques antérieures de niveau 3 pour découvrir et comprendre les contraintes de niveau 2 et leurs conditions de possibilité au niveau 1.

Nous envisageons un autre module pédagogique type (« module de type B ») qui prendrait la forme d'une analyse critique d'une produc-

1 OptimOffice est une chaîne éditoriale qui permet la création de tous types de document. Pour plus de détails, voir : http://scenari-platform.org/projects/optim/fr/ pres/co/ 
tion numérique existante (comme c'est le cas par exemple avec l'analyse d'un texte littéraire en cours de français). Il existe en effet depuis plusieurs décennies des œuvres de création numérique (littérature numérique, art numérique) conçues pour être lues et agies avec un ordinateur. Ces créations numériques, en tant que créations expérimentales, proposent des « cas-limites ». Nous constatons dès lors qu' elles peuvent jouer un rôle de révélateur de tensions entre les différents niveaux du numérique (Bouchardon, 2011). Ces œuvres agissent en effet comme des télescopes à tensions : elles les provoquent et les rendent observables. La mise en valeur des tensions entre les trois niveaux incite à une prise de conscience de l'ensemble des trois niveaux qui pourra ensuite faire l'objet d'un renforcement théorique. Il y aurait donc un enjeu pédagogique à faire travailler les élèves de collèges et de lycées sur des œuvres de littérature et d'art numériques.

En somme, ces quelques pistes didactiques esquissent une diversité des stratégies pédagogiques susceptibles d'être mises en œuvre pour faire acquérir des connaissances méta-scripturales spécifiques à l'écriture numérique et de faciliter leur mobilisation en situation réelle. La complexité du modèle théorique requiert en effet des approches $a d$ $h o c$ en fonction des publics et des notions de niveau 1 et 2 qu'il s'agit de transmettre.

En conclusion, notre approche consiste à avancer qu'il existe des spécificités scripturales propres au support numérique qui méritent d'être enseignées pour développer l'acquisition de connaissances métascripturales tenues pour des ressources nécessaires à la compétence scripturale.

Pour ce faire, nous proposons un modèle heuristique de ces spécificités articulé en trois niveaux : théorique, applicatif et interprétatif. Nous esquissons enfin des pistes pédagogiques qui feront prochainement l'objet d'expérimentations dans le cadre de la seconde phase du projet PRECIP, afin de déterminer dans quelle mesure ce modèle se prête à un enseignement (notamment en termes de niveaux scolaires). Nos recherches à venir auront ainsi pour objectif d'évaluer la possibilité d'adopter une telle approche. Il n'est en effet aucunement évident que la complexité inhérente au modèle puisse faire l'objet d'un enseignement efficace, et ce notamment pour les publics les plus jeunes. Ceci suppose donc un travail de conception pédagogique et de vulgarisation scientifique susceptible de remettre en cause la pertinence pédagogique du modèle, ou tout du moins d'exiger de le faire évoluer. 
Cependant, à l'instar de M. Dabène, nous pensons qu'il existe un enjeu fort à s'approprier des connaissances de l'ordre du « méta » lorsqu'il s'agit de l'écriture, et a fortiori avec les médias numériques. La complexité de ces derniers ne semble pas se limiter à une simple compétence manipulatoire qui, même si elle est nécessaire, ne saurait être suffisante lorsque l'individu « ordinaire » se trouve confronté à un problème technique et/ou communicationnel, et encore moins lorsqu'il doit sans cesse s'adapter à des dispositifs sociotechniques en constante évolution. 


\section{Références}

Barré-de Miniac, C. (2003). Savoir lire et écrire dans une société donnée. Revue française de linguistique appliquée, (2003/1), 107-120.

Bachimont, B. (2000). L'intelligence artificielle comme écriture dynamique : de la raison graphique à la raison computationnelle. In J. Petitot \& P. Fabbri (Ed.), $A u$ nom du sens (pp. 290-319). Paris : Grasset.

Bachimont, B. (2004). Arts et sciences du numérique: Ingénierie des connaissances et critique de la raison computationnelle. Mémoire d'habilitation à diriger les recherches. Université de Technologie de Compiègne.

Bachimont, B. (2007). Ingénierie des connaissances et des contenus. Paris : Hermès Lavoisier.

Bouchardon, S. (2007). L'écriture interactive : une rhétorique de la manipulation. Actes $d u$ colloque H2PTM'07 (pp. 155-170), Hammamet, Tunisie. Paris : Hermès.

Bouchardon, S. (2009). Littérature numérique : le récit interactif. Paris : Hermès science publications.

Bouchardon, S. (2011). Digital Literature and the Digital. Journal of Writing in Creative Practice (JWCP), 4(1), 65-78.

Dabène, M. (1991). Un modèle didactique de la compétence scripturale. Repères. Recherches en didactique du français langue maternelle, 4, 9-22.

Fabre, D., Soudière, M. de L., \& Voisenat, C. (1997). Par écrit. Paris : Éditions MSH.

Jeanneret, Y. (2001). «Informatic literacy : manifestations, captations et déceptions dans le texte informatisé ». Spirale, 28, 11-32.

Goody, J., Bazin, J., \& Bensa, A. (1979). La raison graphique : la domestication de la pensée sauvage. Paris : Minuit.

Perrenoud, P. (1995). Des savoirs aux compétences : de quoi parle-t-on en parlant de compétences ? Pédagogie collégiale, 9(1), 20-24.

Perrenoud, P. (2004a). L'université entre transmission de savoirs et développement de compétences. Girona : Actas del 3er Congrés Internacional : Docència Universitària i Innovación.

Perrenoud, P. (2004b). Évaluer des compétences. Évaluation, 8-11.

Reuter, Y. (2000). Enseigner et apprendre à écrire : construire une didactique de l'écriture. Paris : ESF.

Souchier, E., Jeanneret, Y., \& Le Marec, J. (2003). Lire, écrire, récrire : objets, signes et pratiques des médias informatisés. Paris : BPI.

Souchier, E., \& Jeanneret, Y. (2005). L'énonciation éditoriale dans les écrits d'écran. Communication et langages, 145(1), 3-15. 